\title{
ATP Sulfurylase is Essential for the Utilization of Sulfamate as a Sulfur Source in the Yeast Komagataella pastoris (syn. Pichia pastoris)
}

\author{
Tomas Linder ${ }^{1}$
}

Received: 18 April 2017 / Accepted: 2 June 2017/Published online: 12 June 2017

(c) The Author(s) 2017. This article is an open access publication

\begin{abstract}
The methylotrophic yeast Komagataella pastoris (syn. Pichia pastoris) is one of the few known yeasts that can utilize sulfamate $\left(\mathrm{NH}_{2} \mathrm{SO}_{3}^{-}\right)$as a sulfur source. The biochemical pathway responsible for the catabolism of sulfamate has yet to be identified. The present study sought to investigate whether sulfamate catabolism proceeds through either of the inorganic sulfur intermediates sulfate $\left(\mathrm{SO}_{4}^{2-}\right)$ or sulfite $\left(\mathrm{SO}_{3}^{2-}\right)$ before its assimilation and subsequent incorporation into sulfur-containing amino acids and their derivatives. Two key genes in the $K$. pastoris inorganic sulfur assimilation pathway were deleted separately and the ability of each deletion mutant to utilize sulfamate and other selected sulfur sources was studied. Deletion of the MET3 gene (which encodes the enzyme ATP sulfurylase) did not affect growth on L-methionine, sulfite, methanesulfonate, or taurine but completely abolished growth on sulfate, methyl sulfate and sulfamate. Deletion of the MET5 gene (which encodes the $\beta$ subunit of the enzyme sulfite reductase) abolished growth on all tested sulfur sources except L-methionine. These results suggest that the catabolism of sulfamate proceeds through a sulfate intermediate before its assimilation.
\end{abstract}

\section{Introduction}

Sulfur is an essential element for cellular function and is found in a wide variety of organic biomolecules including the proteinogenic amino acids methionine and cysteine, the

Tomas Linder

tomas.linder@slu.se

1 Department of Molecular Sciences, Swedish University of Agricultural Sciences, Box 7015, 75007 Uppsala, Sweden redox regulator glutathione, the methylation donor $S$ adenosyl methionine and the cofactors biotin, coenzyme A, lipoic acid, and thiamine. The majority of plants and microorganisms can assimilate inorganic forms of sulfur such as sulfate $\left(\mathrm{SO}_{4}^{2-}\right)$, sulfite $\left(\mathrm{SO}_{3}^{2-}\right)$, and sulfide $\left(\mathrm{S}^{2-}\right)$ from their external environment. The conventional inorganic sulfur assimilation pathway is broadly conserved between plants, fungi, bacteria, and archaea with some minor variations between kingdoms [2, 9, 12, 13]. Inorganic sulfur assimilation in yeast and other fungi involves the conversion of sulfate into sulfide via sulfite in four enzymatic steps (Fig. 1). In the first step, intracellular sulfate becomes activated through conjugation to AMP by the enzyme ATP sulfurylase (encoded by the MET3 gene) to form adenylyl sulfate (APS). APS is then converted into phospho-APS (PAPS) by APS kinase (encoded by the MET14 gene). PAPS is subsequently reduced by the enzyme PAPS reductase (encoded by the MET16 gene) to release sulfite. The sulfite that is released from PAPS is reduced into sulfide by sulfite reductase (encoded by the MET5 and MET10 genes). Sulfide can then be incorporated into amino acid pre-cursors and that are subsequently converted into various sulfur-containing biomolecules.

Many microorganisms can also assimilate non-amino acid organosulfur compounds such as sulfoxides, sulfones, sulfonates, and sulfate esters [5, 14], which are abundant in nature [1, 10]. These compounds are first desulfurized to release inorganic sulfur, usually in the form of either sulfate or sulfite [3-5, 14], which can then be assimilated through the inorganic sulfur assimilation pathway. In the common Baker's yeast Saccharomyces cerevisiae, the JLPl gene, which encodes an $\alpha$-ketoglutarate-dependent dioxygenase, is responsible for the desulfurization of sulfonates [3] and possibly sulfate esters as well (Fig. 1). 


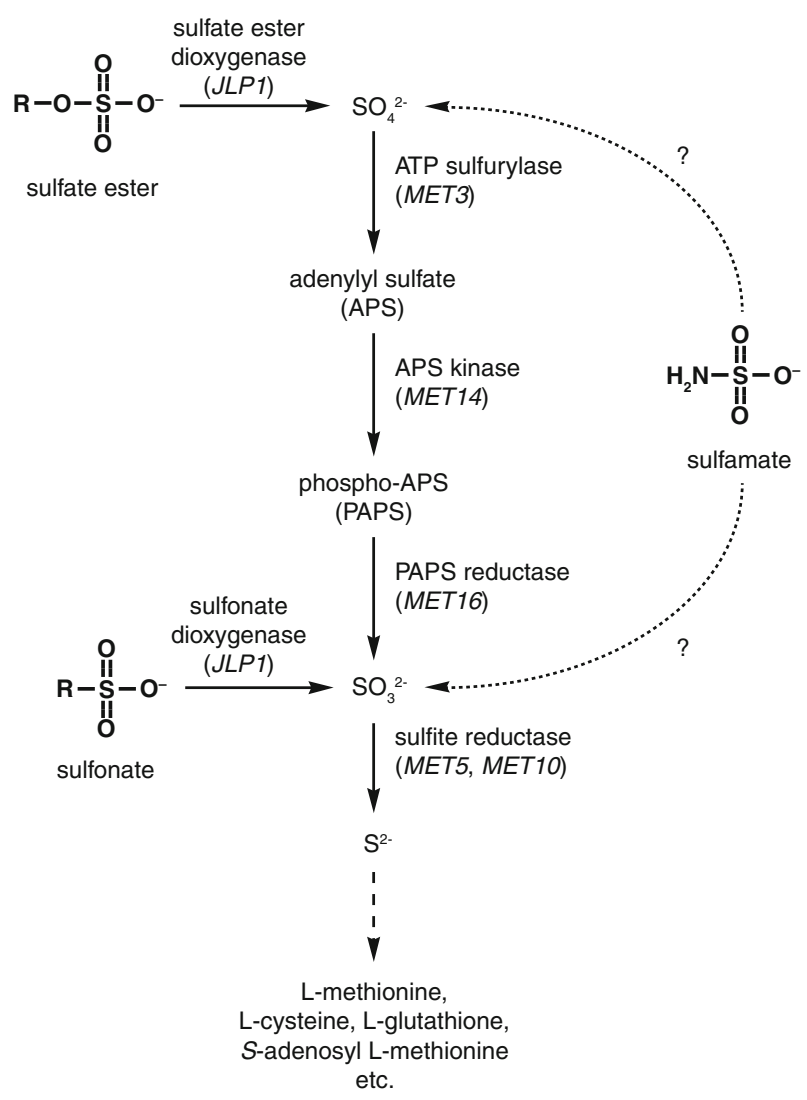

Fig. 1 A simplified overview of the inorganic sulfur assimilation pathway in budding yeasts such as $S$. cerevisiae and $K$. pastoris. Gene names of the corresponding enzymes are shown in brackets

The yeast Komagataella pastoris (syn. Pichia pastoris) is one of a few species of yeast that are known to utilize sulfamate $\left(\mathrm{NH}_{2} \mathrm{SO}_{3}^{-}\right)$as a sulfur source [5]. Nothing is currently known about the catabolic pathway for sulfamate in yeasts but it is likely to involve the cleavage of the $\mathrm{N}-\mathrm{S}$ bond to release either sulfate or sulfite (Fig. 1). The present study sought to investigate whether the inorganic sulfur assimilation pathway is required for the utilization of sulfamate and if so, identify the inorganic sulfur intermediate in $K$. pastoris sulfamate assimilation. This was accomplished through individual deletion of two key genes in the inorganic sulfur assimilation pathway-MET3 and MET5. Phenotypical analysis of both mutants on sulfamate as well as selected reference sulfur compounds was then used to identify the likely inorganic sulfur intermediate.

\section{Materials and Methods}

\section{Yeast Integration Constructs}

The intergenic regions (IGRs) flanking the $K$. pastoris MET3 and MET5 ORFs were amplified and inserted into the pFA6a-kanMX4 plasmid (GenBank accession AJ002680, [15]) to enable targeted gene replacement. The KpMET3 5' IGR (GenBank accession CABH01000262, residues 20,406-20,990) was amplified from $K$. pastoris CBS 704 genomic DNA with primers KPMET3 $5^{\prime}$ fwd (GCG CGC CCC GGG ATT TAA ATG CCG AAA GAT TCA A) and KpMET3 $5^{\prime}$ rev (GCG CGC GGA TCC TTG AGA GAT CTT TCA CTG), digested with BamHI and $S m a \mathrm{I}$, and inserted into $B g l \mathrm{II} / S m a \mathrm{I}$-cut pFA6a-kanMX4 to produce the intermediate plasmid pFA6a-KpMET3_5'kanMX4. The KpMET3 $3^{\prime}$ IGR (GenBank accession CABH01000262, residues 18,241-18,734) was amplified from $K$. pastoris CBS 704 genomic DNA with primers KpMET3 $3^{\prime}$ fwd (GCG CGC GGA TCC ATA GTA GAC TTT GTA ATG) and KpMET3 $3^{\prime}$ rev (GCG CGC ATT TAA ATA ATG AGT ATG TTA TC), digested with BamHI and SwaI and inserted into BamHI/SwaI-cut pFA6a-KpMET3_5'-kanMX4 to produce the plasmid pFA6a-Kp_Amet3-kanMX4 (Fig. 2a).

The KpMET5 5' IGR (GenBank accession CABH 01000235, residues 383,892-384,453) was amplified from K. pastoris CBS 704 genomic DNA with primers KpMET5 $5^{\prime}$ fwd (GCG CGC CCC GGG ATT TAA ATT ATG GAT TAT GTA A) and KpMET5 $5^{\prime}$ rev (GCG CGC AGA TCT TCA ACC AAC AGA ACC GGT CAT), digested with $B g l \mathrm{II}$ and $S m a \mathrm{I}$ and inserted into $B g l \mathrm{II} / S m a \mathrm{I}$-cut pFA6akanMX4 to produce the intermediate plasmid pFA6aKpMET5_5'-kanMX4. The KpMET5 3' IGR (GenBank accession CABH01000235, residues 379,251-379,682) was amplified from $K$. pastoris CBS 704 genomic DNA with primers KpMET5 $3^{\prime}$ fwd (GCG CGC GGA TCC GAA GAA GCC ATT GCA TAG) and KpMET5 $3^{\prime}$ rev (GCG CGC ATT TAA ATG TAA CGT AAC CTT A), digested with BamHI and SwaI and inserted into BamHI/SwaI-cut pFA6a-KpMET5_5'-kanMX4 to produce the plasmid pFA6a-Kp_Amet5-kanMX4 (Fig. 2a). Prior to transformation the pFA6a-Kp_Amet3-kanMX4 and pFA6a$K p \_\Delta m e t 5-k a n M X 4$ plasmids were linearized through digestion with $S w a \mathrm{I}$ (Fig. 2b) and purified into sterile water using the QIAquick PCR purification kit (Qiagen).

\section{Yeast Transformation}

The parent yeast strain for the transformations in this study was $K$. pastoris CBS 704, which was purchased from Centraalbureau voor Schimmelcultures (Utrecht, the Netherlands). The selection agent G418 disulfate was purchased from Formedium Ltd. (Norfolk, UK). An aqueous stock solution of G418 disulfate was prepared to a final concentration of $100 \mathrm{~g} \mathrm{l}^{-1}$, sterilized by filtration and stored as aliquots at $-20{ }^{\circ} \mathrm{C}$. The transformation protocol used in this study has been described previously [6]. Following transformation, cells were plated out on selective 
a

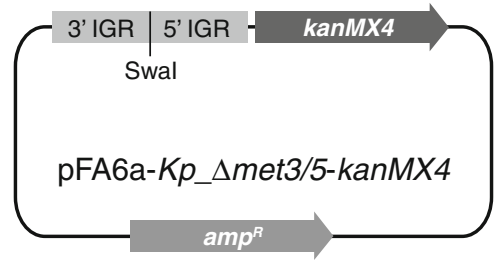

C

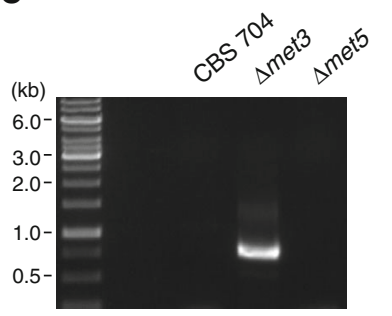

d

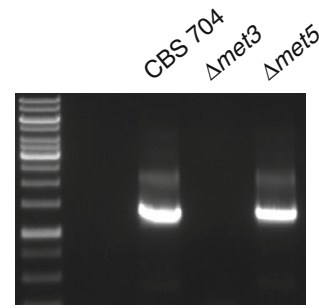

Fig. 2 Plasmid design and integration of plasmid constructs at the $K$. pastoris MET3 and MET5 loci. a Design of the MET3 and MET5 integration plasmids. The $K p_{-} \_$met 3 and $K p_{-} \_$Amet5 targeting cassettes were assembled in the pFA6a-kanMX4 plasmid to produce plasmids pFA6a-Kp_Amet3-kanMX4 and pFA6a-Kp_Amet5-kanMX4, respectively. Plasmid components are not drawn to scale. b The plasmids pFA6a-Kp_Amet3-kanMX4 and pFA6a-Kp_Amet5-kanMX4 were linearized by digestion with $S w a$ I to enable homologous recombination with the $5^{\prime}$ and $3^{\prime}$ IGRs of MET3 and MET5. The locations of control primers to confirm correct integration of the construct are indicated. DNA elements are not drawn to scale.

media consisting of YMPD agar $\left(3 \mathrm{~g}\right.$ yeast extract $1^{-1}, 3 \mathrm{~g}$ malt extract $1^{-1}, 5 \mathrm{~g}$ peptone $1^{-1}, 10 \mathrm{~g}$ glucose $1^{-1}, 20 \mathrm{~g}$ agar $1^{-1}$ ) with $400 \mathrm{mg} \mathrm{G} 418$ disulfate $1^{-1}$. Correct chromosomal integration and deletion of the MET3 and MET5 loci were confirmed by PCR analysis of purified genomic DNA from each strain (Fig. 2c-f). Correct integration at the MET3 locus was assayed using primers pFA6a ctrl fwd (ACT GAG AGT GCA CCA TAT GGA) and KpMET3 $3^{\prime}$ ctrl rev (TCG GTT GTC AGA TGG CA), which produce no product in the CBS 704 parent strain and the 4 met5 strain but a 715-bp amplification product in the $4 m e t 3$ strain (Fig. 2c). Successful deletion of the MET3 gene was assayed using primers KpMET3 ctrl fwd (TAC GCT CCA ATT GAC ACA GTC) and KpMET3 $3^{\prime}$ ctrl rev, which produce a 1199-bp amplification product in the CBS 704 parent strain and the $\Delta$ met 5 strain but no product in the $\triangle m e t 3$ strain (Fig. 2d). Correct integration at the MET5 locus was assayed using primers pFA6a ctrl fwd and KpMET5 $3^{\prime}$ ctrl rev (TCT CGA GAC ACA CAT CTG), which produce no product in the CBS 704 parent strain and the $\Delta m e t 3$ strain but a 643-bp amplification product in the $\triangle m e t 5$ strain (Fig. 2e). Successful deletion of the MET5 gene was assayed using primers KpMET5 ctrl fwd (TCA TAA GAC TGG TCT CCT GGA) and KpMET5 $3^{\prime}$ ctrl rev, which produce a 1107-bp amplification product in the CBS e

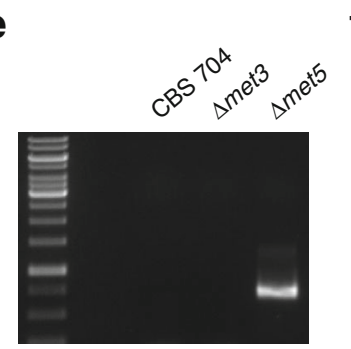

$\mathbf{f}$

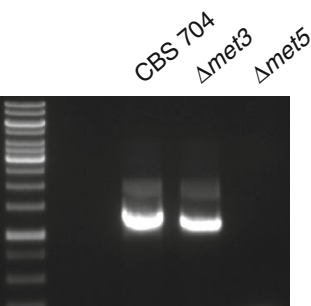

c Confirmation of the correct integration of the linearized pFA6aKp_Amet3-kanMX4 construct as demonstrated by PCR of genomic DNA using primers pFA6a fwd and KpMET3 $3^{\prime}$ ctrl rev. d Confirmation of the removal of the endogenous MET3 locus as demonstrated by PCR of genomic DNA using primers KpMET3 ctrl fwd and KPMET3 $3^{\prime}$ ctrl rev. e Confirmation of the correct integration of the linearized pFA6a-Kp_Amet5-kanMX4 construct as demonstrated by PCR of genomic DNA using primers pFA6a fwd and KpMET5 $3^{\prime} \mathrm{ctrl}$ rev. $\mathbf{f}$ Confirmation of the removal of the endogenous MET5 locus as demonstrated by PCR of genomic DNA using primers KpMET5 ctrl fwd and KPMET5 $3^{\prime}$ ctrl rev

704 parent strain and the $4 m e t 3$ strain but no product in the $\Delta m e t 5$ strain (Fig. 2f).

\section{Sulfur Utilization Assays}

A sulfur-limited glucose medium (SLD) with only trace amounts of sulfate $(\leq 6 \mu \mathrm{M})$ was used for assaying growth on individual sulfur-containing compounds [5]. SLD medium consisted of $1.2 \mathrm{~g}$ yeast nitrogen base without amino acids, ammonium sulfate, or magnesium $1^{-1}$ (Formedium Ltd., Norfolk, UK), $4 \mathrm{~g}$ ammonium chloride $\mathrm{l}^{-1}$, $0.84 \mathrm{~g}$ magnesium chloride hexahydrate $\mathrm{l}^{-1}$, and $20 \mathrm{~g}$ glucose $1^{-1}$. Prior to the sulfur utilization assay, each yeast strain was pre-cultured in $3 \mathrm{ml}$ methionine-supplemented minimal glucose medium consisting of $6.7 \mathrm{~g}$ Difco yeast nitrogen base without amino acids $1^{-1}$ (Becton, Dickinson and Company), $20 \mathrm{~g}$ glucose $1^{-1}$, and $1 \mathrm{mM}$ L-methionine. Pre-cultures were washed twice in sterile deionized water before being re-suspended in $2.97 \mathrm{ml} \mathrm{SLD}$ in a 50-ml tube to a final optical density at $600 \mathrm{~nm}\left(\mathrm{OD}_{600}\right)$ of 0.005 . Individual sulfur-containing compounds were added as $30 \mu \mathrm{l}$ of a $10 \mathrm{mM}$ stock solution, making a final concentration of $0.1 \mathrm{mM}$. A non-supplemented sample with $30 \mu \mathrm{l}$ deionized water was used as a control. Chloramphenicol $\left(15 \mathrm{mg} \mathrm{l}^{-1}\right) \quad$ was included to prevent bacterial 
contamination. Incubations were carried out at $30{ }^{\circ} \mathrm{C}$ in a rotary shaker set to $200 \mathrm{rpm}$. Growth was monitored by measurement of $\mathrm{OD}_{600}$ after 6 days using an Ultrospec 1100 pro spectrophotometer with a $1 \mathrm{~cm}$ pathlength (GE Healthcare). Each sulfur utilization assay was carried out in triplicate.

\section{Results and Discussion}

The inorganic sulfur assimilation pathway is required both for the assimilation of inorganic sulfur as well as nonamino acid organosulfur compounds such as sulfonates and sulfate esters (Fig. 1). Desulfurization of sulfonates and sulfate esters has not been previously studied in $K$. pastoris but its genome contains two genes (PIPA01971 and PIPA02153) that encode putative $\alpha$-ketoglutarate-dependent sulfonate/sulfate ester dioxygenases homologous to the $S$. cerevisiae JLP1 gene [3] and the bacterial atsK gene [4].

ATP sulfurylase (encoded by the gene MET3) and sulfite reductase (encoded by the genes MET5 and MET10) are key enzymes in the inorganic sulfur assimilation pathway and deletion of either gene can demonstrate whether the assimilation of a particular sulfur compound proceeds through a sulfate or sulfite intermediate. Deletion of MET3 is expected to prevent growth on sulfur sources assimilated through a sulfate intermediate (such as sulfate esters) but not those assimilated through a sulfite intermediate (such as sulfonates), while deletion of MET5 is expected to prevent growth on sulfur sources assimilated through either a sulfate or sulfite intermediate. The present study therefore sought to gain a better understanding of sulfamate catabolism through phenotypical assays of MET3 and MET5 deletion mutants cultivated on minimal medium containing a single sulfur source. In addition to sulfamate, six additional sulfur sources were included for reference. These included the sodium salts of sulfate and sulfite, the sulfate ester methylsulfate and the two sulfonates taurine and methanesulfonate. L-Methionine was included as a reference as its assimilation does not require the inorganic sulfur assimilation pathway.

The K. pastoris MET3 and MET5 genes were deleted individually by homologous gene replacement using the kanMX4 G418 disulfate resistance marker (Fig. 2b). Neither mutant displayed any detectable growth defect on rich YMPD medium but failed to show any detectable growth on a minimal medium where sulfate was the only available sulfur source (data not shown). Growth was restored in both mutants if the minimal medium was supplemented with L-methionine. Both mutants were subsequently assayed for growth on chemically defined sulfur-limited medium (SLD) supplemented with different organic or inorganic sources of sulfur to final concentration of $0.1 \mathrm{mM}$ total sulfur. (Neither mutant grew in SLD medium lacking sulfur supplementation.) Growth was measured 6 days after inoculation. The parent strain CBS 704 was included as a reference.

As expected neither mutant displayed any detectable growth on sulfate as the only sulfur source after 6 days, while only the $\Delta$ met 3 mutant could grow when sulfite was the only available sulfur source (Fig. 3). Both mutants grew well on L-methionine comparable to the CBS 704 parent strain. The MET3 gene appeared to be entirely dispensable for growth when L-methionine, taurine, or methanesulfonate was the only available sulfur source, which was expected as the assimilation of these sulfur sources does not involve a sulfate intermediate. The $\Delta m e t 3$ strain did not grow on methyl sulfate, which agrees with previous work that has shown that the assimilation of sulfate esters proceeds through a sulfate intermediate [4]. Notably, neither mutant could grow when sulfamate was the only available sulfur source, which demonstrates the requirement for the inorganic sulfur assimilation pathway in sulfamate assimilation and also suggests that sulfamate assimilation in $K$. pastoris proceeds through a sulfate intermediate.

Deletion of the MET5 gene abolished growth for all sulfur sources with the exception of L-methionine (Fig. 3). This result was expected for sulfur sources with an absolute requirement for the MET3 gene (which will consequently not grow in the absence of MET5 gene either) as well as sulfur compounds known or predicted to be assimilated through a sulfite intermediate such as sulfonates (taurine and methanesulfonate).

In summary, this study has demonstrated for the first time the requirement for the MET3 gene for the utilization of sulfamate as a sole source of sulfur in yeast. This observation suggests that the catabolism of sulfamate produces sulfate rather than sulfite as an intermediate before assimilation in $K$. pastoris and possibly other

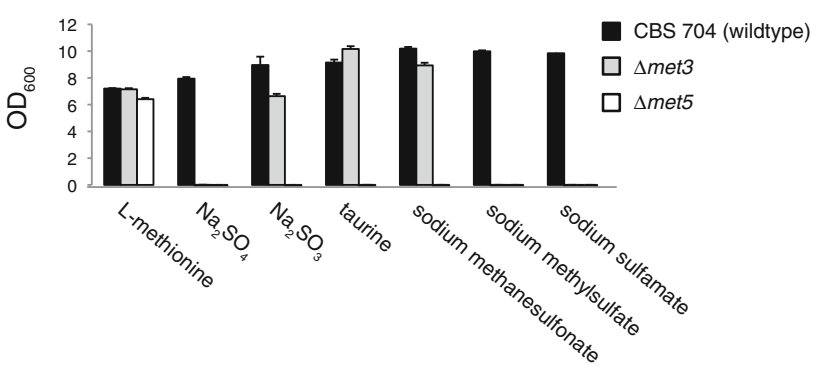

Fig. 3 Growth of $K$. pastoris deletion mutants on selected sulfur sources. Bars represent the average optical density $\left(\mathrm{OD}_{600}\right)$ value after 6 days incubation in $3 \mathrm{ml}$ chemically defined medium containing $0.1 \mathrm{mM}$ of the indicated sulfur source. Growth assays were carried out in triplicate with error bars indicating one standard deviation 
sulfamate-utilizing yeasts as well. The enzymatic activity responsible for sulfamate catabolism in $K$. pastoris still remains to be identified. Sulfamate compounds are known to occur in nature, the most predominant form being $N$ sulfated glucosamine residues in the linear glycosaminoglycans heparin and heparan sulfate. Sulfamate functional groups have also been described in a number of natural products [7]. Two enzymes belonging to the arylsulfatase protein family (Pfam accession number PF00884) from human [11] and the bacterium Pedobacter heparinus [8] have been shown to desulfurize sulfamate groups in heparan sulfate. Genes encoding proteins belonging to the arylsulfatase family are present in a number of yeasts, including well-characterized species such as Kluyveromyces lactis, Schizosaccharomyces pombe, and Yarrowia lipolytica. However, previous studies have shown no correlation between the presence of putative arylsulfatases in these species and the ability to utilize sulfamate as a sulfur source [5]. The $K$. pastoris CBS 704 genome lacks any detectable arylsulfatase homologs, which further argues against a role for this protein family in sulfamatase utilization in yeast. The significant evolutionary distance between $K$. pastoris and $S$. cerevisiae as well as the greater metabolic versatility of $K$. pastoris with respect to sulfur makes this yeast a promising system for greater understanding of the metabolic pathways, involved in the assimilation of alternative sulfur sources in yeast.

\section{Compliance with Ethical Standards}

Conflict of interest The author declares no conflict of interest.

Human and Animal Rights Informed Consent This article does not contain any studies with human participants or animals performed by the author.

Open Access This article is distributed under the terms of the Creative Commons Attribution 4.0 International License (http://crea tivecommons.org/licenses/by/4.0/), which permits unrestricted use, distribution, and reproduction in any medium, provided you give appropriate credit to the original author(s) and the source, provide a link to the Creative Commons license, and indicate if changes were made.

\section{References}

1. Autry AR, Fitzgerald JW (1990) Sulfonate-S-a major form of forest soil organic sulfur. Biol Fertil Soils 10:50-56

2. Hébert A, Casaregola S, Beckerich JM (2011) Biodiversity in sulfur metabolism in hemiascomycetous yeasts. FEMS Yeast Res 11:366-378

3. Hogan DA, Auchtung TA, Hausinger RP (1999) Cloning and characterization of a sulfonate/alpha-ketoglutarate dioxygenase from Saccharomyces cerevisiae. J Bacteriol 181:5876-5879

4. Kahnert A, Kertesz MA (2000) Characterization of a sulfurregulated oxygenative alkylsulfatase from Pseudomonas putida S-313. J Biol Chem 275:31661-31667

5. Linder T (2012) Genomics of alternative sulfur utilisation in ascomycetous yeasts. Microbiology 158:2585-2597

6. Linder T (2014) CMO1 encodes a putative choline monooxygenase and is required for the utilization of choline as the sole nitrogen source in the yeast Scheffersomyces stipitis (syn. Pichia stipitis). Microbiology 160:929-940

7. Mujumdar P, Poulsen SA (2015) Natural product primary sulfonamides and primary sulfamates. J Nat Prod 78:1470-1477

8. Myette JR, Soundararajan V, Behr J, Shriver Z, Raman R, Sasisekharan R (2009) Heparin/heparan sulfate $N$-sulfamidase from Flavobacterium heparinum: structural and biochemical investigation of catalytic nitrogen-sulfur bond cleavage. J Biol Chem 284:35189-35200

9. Saito K (2004) Sulfur assimilatory metabolism. The long and smelling road. Plant Physiol 136:2443-2450

10. Schäfer H, Myronova N, Boden R (2010) Microbial degradation of dimethylsulphide and related $\mathrm{C}_{1}$-sulphur compounds: organisms and pathways controlling fluxes of sulphur in the biosphere. J Exp Bot 61:315-334

11. Scott HS, Blanch L, Guo XH, Freeman C, Orsborn A, Baker E, Sutherland GR, Morris CP, Hopwood JJ (1995) Cloning of the sulphamidase gene and identification of mutations in Sanfilippo A syndrome. Nat Genet 11:465-467

12. Sekowska A, Kung HF, Danchin A (2000) Sulfur metabolism in Escherichia coli and related bacteria: facts and fiction. J Mol Microbiol Biotechnol 2:145-177

13. Thomas D, Surdin-Kerjan Y (1997) Metabolism of sulfur amino acids in Saccharomyces cerevisiae. Microbiol Mol Biol Rev 61:503-532

14. Uria-Nickelsen MR, Leadbetter ER, Godchaux W (1993) Sulfonate-sulfur assimilation by yeasts resembles that of bacteria. FEMS Microbiol Lett 114:73-77

15. Wach A, Brachat A, Pohlmann R, Philippsen P (1994) New heterologous modules for classical or PCR-based gene disruptions in Saccharomyces cerevisiae. Yeast 10:1793-1808 